\title{
Intermédialités
}

Histoire et théorie des arts, des lettres et des techniques

Intermediality

History and Theory of the Arts, Literature and Technologies

\section{Exposer le temps}

\section{Véronique Souben et Elitza Dulguerova}

Numéro 15, printemps 2010

exposer

displaying

URI : https://id.erudit.org/iderudit/044678ar

DOI : https://doi.org/10.7202/044678ar

Aller au sommaire du numéro

Éditeur(s)

Revue Intermédialités (Presses de l’Université de Montréal)

ISSN

1705-8546 (imprimé)

1920-3136 (numérique)

Découvrir la revue

Citer ce document

Souben, V. \& Dulguerova, E. (2010). Exposer le temps. Intermédialités /

Intermediality, (15), 119-141. https://doi.org/10.7202/044678ar
Résumé de l'article

Le point de départ de ce dialogue est l'exposition 90', conçue et organisée par Véronique Souben au Frac Franche-Comté en 2009. Son dispositif scénographique très rigoureux, articulé en dix salles, déclinait autant de conventions expositionnelles, de la galerie chronologique à la period room, de l'accrochage en tapisserie au modèle du white cube, du plateau cinématographique aux halls d'entrée. En situant cette expérience dans l'histoire des formes d'exposition artistiques autant que commerciales du $19^{\mathrm{e}}$ et $d u 20^{\mathrm{e}}$ siècle, les auteures réfléchissent aux rapports entre exposition et collection, au rôle du commissaire, à la place du catalogue, et mettent en évidence le travail de représentation du temps et de l'histoire que produit une exposition. 


\title{
Exposer le temps
}

\author{
Véronique Souben \\ en dialogue avec Elitza Dulguerova
}

\begin{abstract}
u 18 juin au 4 octobre 2009, le Fonds régional d'art contemporain de Franche-Comté a accueilli l'exposition 9o', conçue par la commissaire indépendante Véronique Souben en vue de présenter l'ensemble des acquisitions du Frac depuis son ouverture en 1982. Historienne de l'art spécialisée dans le design, Véronique Souben étudie plus particulièrement les liens entre le domaine des arts plastiques et du design à travers, notamment, leurs modes de présentation. Le projet 9o' met en place (et en acte) une réflexion qui porte à la fois sur la notion même de «collection » et sur les possibilités de son «exposition ».
\end{abstract}

Elitza Dulguerova: Comme son titre l'indique, l'exposition 9o' que vous avez organisée en 2009 pour le Frac Franche-Comté met l'accent sur la part importante de la temporalité dans l'expérience que nous faisons d'une ouvre, voire d'un ensemble d'œuvres d'art. Quels partis pris ont motivé ce concept directeur?

Véronique Souben: Ce titre reflétait mon désir de réaliser un projet en rupture avec l'ensemble des expositions que j'ai coutume de voir et qui ont un format assez similaire, une approche thématique ou esthétique réglée autour d'accrochages extrêmement homogènes. Dans la majeure partie des cas, les expositions sont réalisées pour «servir» les œuvres d'art, les mettre en valeur. La structure sacralisante du white cube $e^{1}$ sur laquelle s'alignent un grand nombre d'institutions aide en cela. Mais elle a fini par enfermer les œuvres dans un cadre idéologique dont les visées paraissent souvent plus commerciales qu’esthétiques.

1. Terme initié par Brian O'Doherty dans son livre Inside the White Cube. The Ideology of the Gallery Space, Santa Monica et San Francisco, The Lapis Press, 1976; en français: White Cube. L'espace de la galerie et son idéologie, trad. Catherine Vasseur, revue par Patricia Falguières, Zurich, JRP-Ringier, 2008. 
J'ai voulu renverser ce principe en imaginant une exposition dont le but ne serait plus de mettre en avant l'œuvre mais le visiteur, à travers son temps. Quant au titre, la durée de 90 minutes me semblait correspondre au temps moyen que consacre un visiteur quelconque à la visite d'un musée en France ou en Europe. Les résultats d'un sondage du ministère de la Culture ont ensuite corroboré cette intuition: sur une période de dix années, les temps moyens de visite de cent un musées français, du plus gros (Orsay, Le Louvre) au plus modeste, atteignaient précisément la moyenne générale de... 90 minutes!

Réfléchir à l'exposition à partir de la notion de temps me semblait être une approche pas seulement originale ou expérimentale mais nécessaire. Le temps, omniprésent et toujours plus fragmenté, régit et conditionne de manière diffuse mais réelle l'ensemble de nos activités, que ce soit au travail ou pendant nos «temps libres». La moindre de nos escapades culturelles est soumise à un contrôle temporel de plus en plus serré. Ce temps est d'autant plus contrôlé que le nombre de visiteurs, dans certaines institutions, a augmenté notablement en quelques années. De nouveaux impératifs de régulation des flux s'imposent pour des raisons économiques de rentabilité. Et pourtant, à ma connaissance, peu de commissaires développent une pensée critique sur le sens et la portée idéologique du parcours.

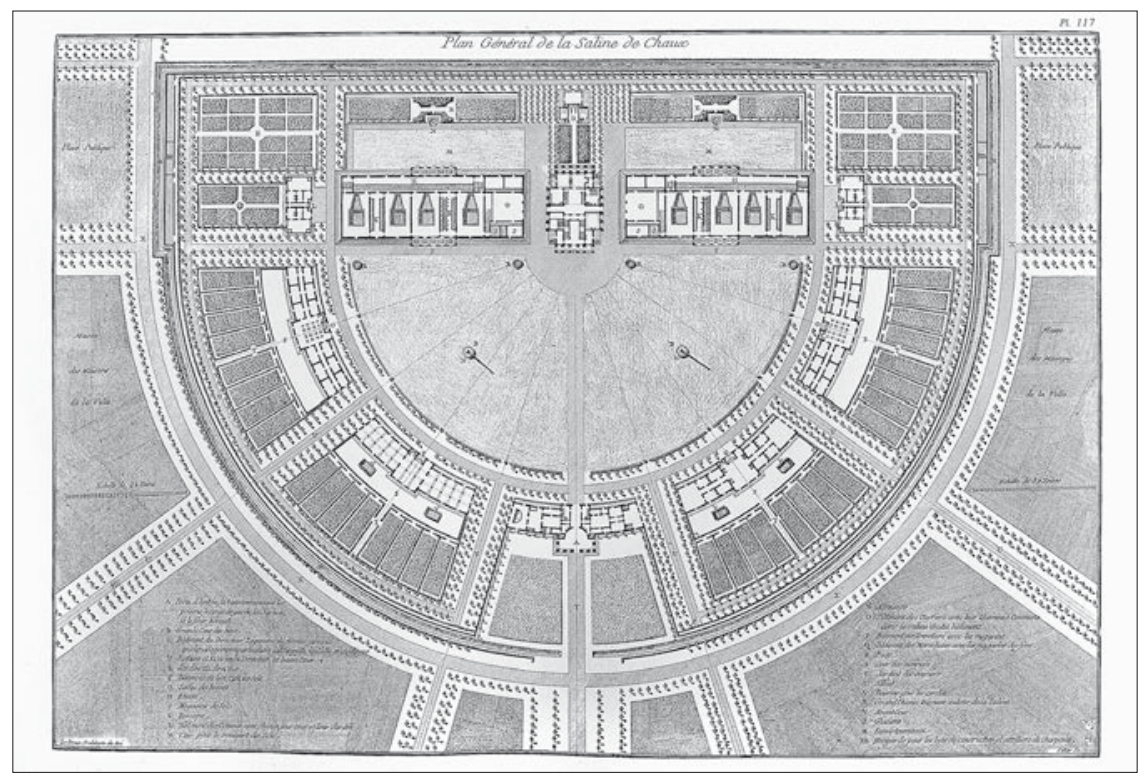

Fig. 1: Claude Nicolas Ledoux, plan général de la Saline Royale d'Arc-et-Senans, extrait de Larchitecture considérée sous le rapport des arts, des mours et de la législation, planche 16, Paris, Chez l'auteur, rue neuve d'Orléans, 1814, (c) Photothèque EPCC Saline royale. 


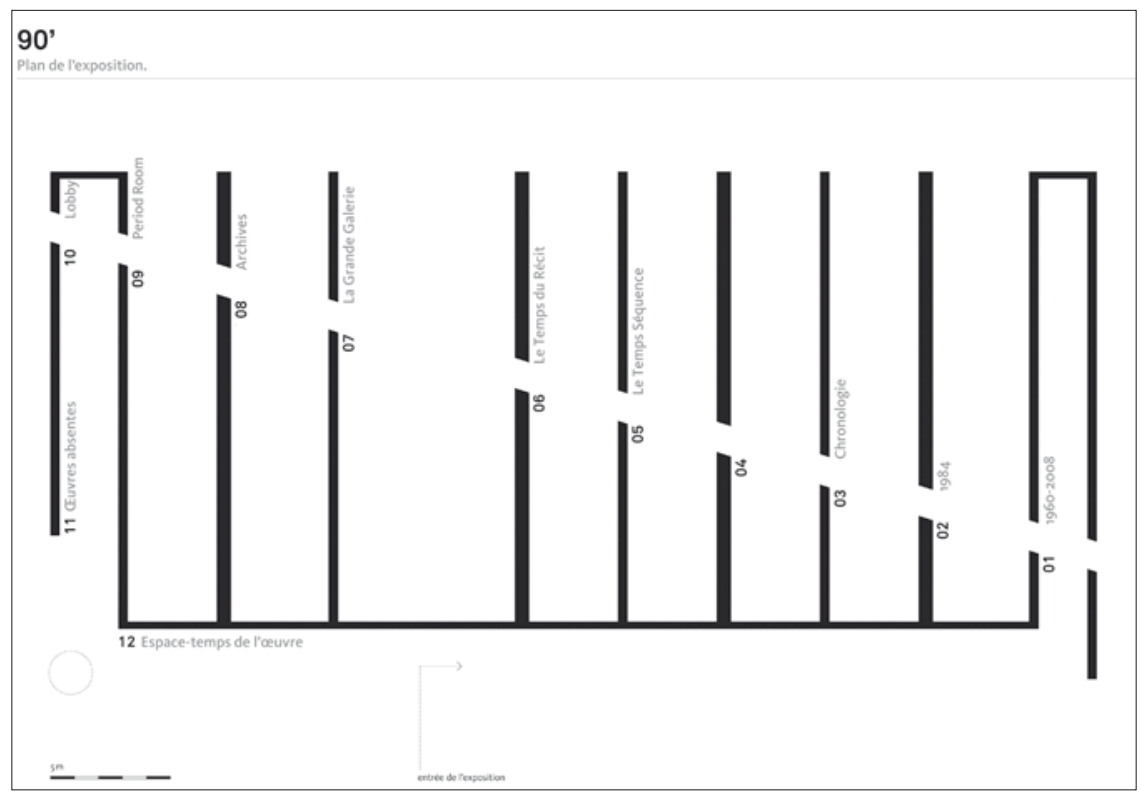

Fig. 2: Plan de l'exposition 9o', commissaire Véronique Souben, Frac Franche-Comté, 18 juin au 4 octobre 2009 .

Ce sont, entre autres, ces observations qui m’ont amenée à concevoir un projet autour du visiteur, de son temps et de son parcours. Le hasard voulait également que la ville de Besançon et la région Franche-Comté où devait se tenir l'exposition étaient le «berceau » du temps. C'est en effet là qu'aux $18^{\text {e }}$ $19^{\mathrm{e}}$ siècles résidait toute l'industrie horlogère, avant que la Suisse ne prenne le relais. Dans ce contexte géographique, le temps avait une valeur culturelle extrêmement forte.

L'autre facteur déterminant fut le lieu dans lequel l'exposition devait prendre place: la Saline Royale d'Arc-et-Senans. Il s'agit d'une structure industrielle étonnante conçue au $18^{\mathrm{e}}$ siècle par le grand architecte "utopiste » et visionnaire Claude-Nicolas Ledoux (fig. 1). Dessiné sur la base d'un arc de cercle parfait, ce complexe architectural unique en son genre devait refléter l'utopie sociale de travail et de vie que Ledoux préconisait. Il me semblait intéressant de faire correspondre la fonctionnalité économique et sociale de la Saline avec la fonctionnalité du parcours de l'exposition 9o'. Cette correspondance prit la forme d'un dispositif qui se voulait aussi rigoureux, systématique et fonctionnel que l'est l'architecture de Ledoux (fig. 2). Pour souligner ce parti pris autoritaire, j'avais installé au début et à la fin du parcours deux grandes horloges qui affichaient 
en boucle l'écoulement de ce laps de temps. Elles étaient disposées de telle sorte qu'il était difficile, pour le visiteur, d'y échapper. Or, si leur numérotation semblait imposer un contrôle, dans la pratique celle-ci ne régulait rien, mais elle rappelait aux visiteurs l'incidence du temps sur leur parcours.

\section{SCÉNOGRAPHIE ET PARCOURS SPATIAL}

Le parti pris de centrer l'exposition sur le spectateur et son temps de visite évoque la dialectique, au sein des projets des avant-gardes historiques, entre recherche de mobilité et contrôle du parcours. El Lissitzky, par exemple, espérait réaliser l'utopie d'un visiteur actif qui manipule des dispositifs de présentation des ceuvres pour en faire une expérience multisensorielle ${ }^{2}$. Quant à Herbert Bayer, ses projets pour le Bauhaus et pour le MoMA à New York étaient très ambivalents: d'une part, il optait pour des plans d'exposition non rectilignes, biomorphes, dont les courbes dessinaient des espaces de déplacement fluides et continus; mais simultanément, une signalisation marquée à même le sol indiquait au visiteur les directions et parcours préférés et les points de vue privilégiés.

Dans le plan qui accompagne l'exposition 9o', le dispositif spatial dessine un parcours directeur très clair, voire dirigiste. Est-ce qu'il était conçu pour gérer le parcours du visiteur et si oui, comment?

Le dispositif est devenu le sujet ou, plus concrètement, l'objet même de 9o'. Il faut préciser que cette exposition fut conçue en réponse à un appel à projets lancé par le Frac Franche-Comté en 2008, dont la contrainte principale résidait dans le pourcentage d'œuvres qu'il fallait exposer: $80 \%$ des 463 «expôts » que regroupe la collection du Frac, parmi lesquels des installations, des films...

Le dispositif représentait donc le point de départ à partir duquel j’allais pouvoir présenter l'ensemble de la collection tout en «mettant en scène » diverses temporalités. Le lieu dans lequel il devait être édifié était le Bâtiment du Sel: une grande boîte rectangulaire de $1200 \mathrm{~m}^{2}$ tenue par des colonnes en béton. À l'image des bassins parallèles dans lesquels étaient à l'époque réparties les tonnes

2. À ce sujet, voir Yves-Alain Bois, «Exposition: esthétique de la distraction, espace de démonstration", Les cahiers du Musée national d'art moderne, n 29 "En revenant de l'exposition", automne 1989, p. 57-79; Maria Gough, "Constructivism Disoriented: El Lissitzky's Dresden and Hanover Demonstrationsräume», dans Nancy Perloff et Brian Reed (dir.), Situating El Lissitzky, Los Angeles, Getty Research Institute, 2003, p. 77-125.

3. Voir Mary-Anne Staniszewski, The Power of Display. A History of Exhibition Installations at the Museum of Modern Art, Cambridge (Mass.) et Londres, MIT Press, 1998. 
de sel à traiter, le dispositif que j’ai imaginé consistait en une série de dix galeries parallèles. La longueur et l'étroitesse effectives de ces galeries (dix-huit mètres de long sur trois à quatre mètres de large) les apparentaient à des couloirs. Ce rapprochement était primordial afin d'éviter toute similitude avec le cube, l'espace clos ou la cellule-habitacle. Dans la pratique, ces galeries-couloirs ouvertes sur le côté nord et fermées côté sud, sans parois intermédiaires, sans obstacle - si ce n'est parfois les œuvres -, avaient pour but de favoriser une déambulation fluide et continue.

Lautre élément déterminant dans ce dispositif était la diagonale qui traversait d'un bout à l'autre ce grand rectangle composé de dix galeries (fig. 3). Elle avait deux fonctions: en tant que perspective, elle donnait au visiteur l'impression de maitriser tout l'espace, donnant à voir l'enfilade de toutes les galeries. En tant que passage, elle lui permettait de traverser l'exposition d'une seule traite, sans arrêt. Cette conception du parcours différencié pouvait rappeler celle que Le Corbusier avait imaginée pour le Musée d'Art moderne de la ville de Paris en $1937^{4}$. Le projet architectural - malheureusement non retenu - de Le Corbusier

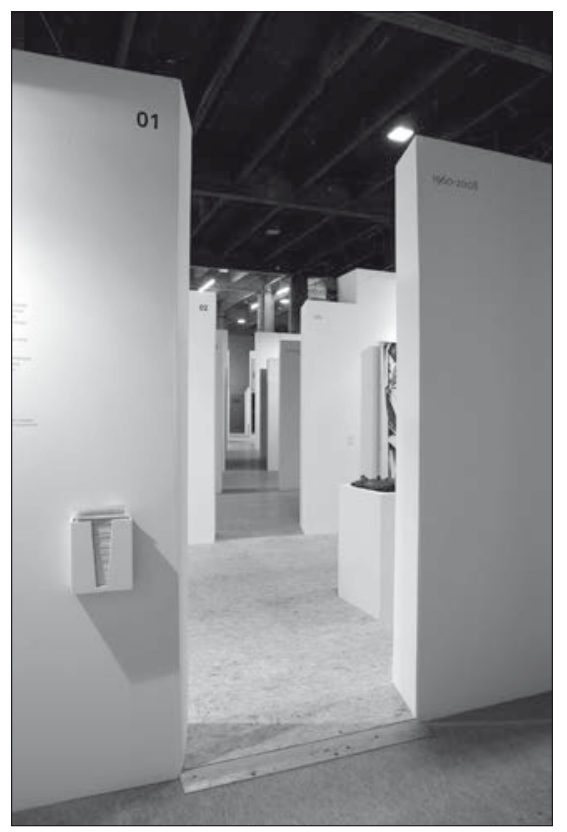

Fig. 3: 9o', vue de la diagonale traversant l'espace d'exposition. Photographie Aurélien Mole.

4. Voir Max Bill (dir.), Le Corbusier \& P. Jeanneret. Euvre complète 1934-1938, Zurich, Les Éditions d'Architecture, 1964, p. 87. 


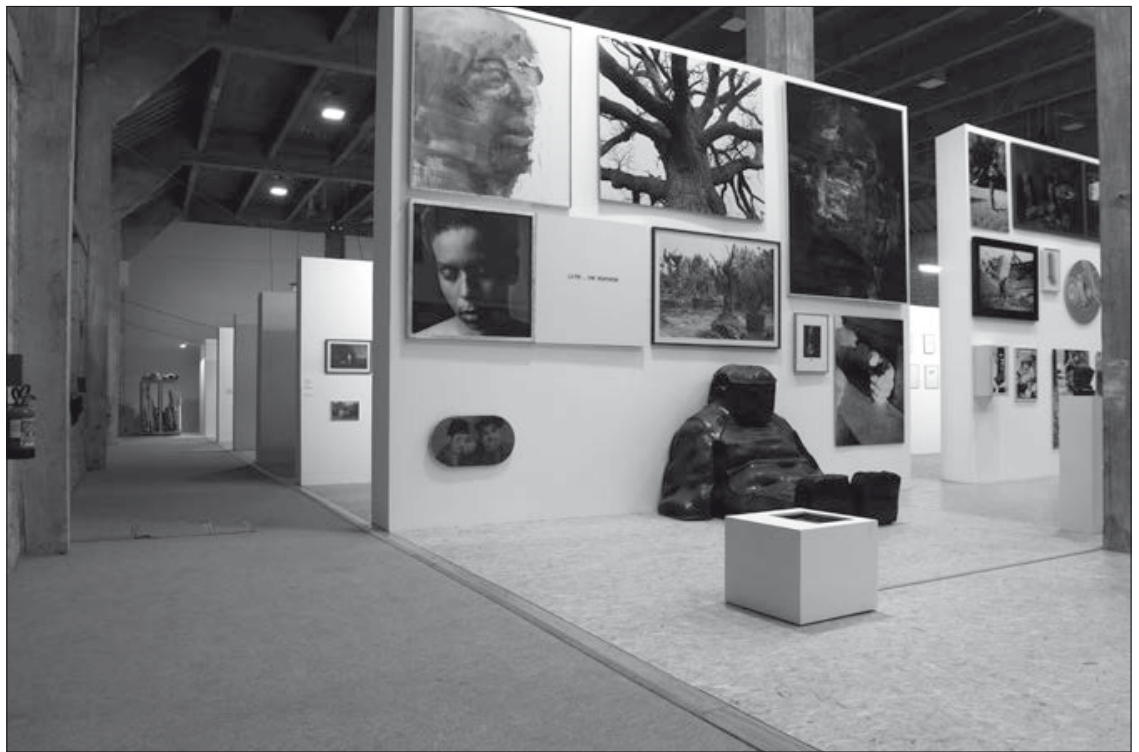

Fig. 4: 9o', vue du côté nord et détail de La Grande Galerie. Photographie Aurélien Mole.

plaçait le visiteur au centre même du projet architectural à travers trois types de cheminement différents: le premier destiné aux touristes, le deuxième aux esthètes et le troisième aux spécialistes. D’une certaine manière, le projet 9o' est assez proche de ces «balades architecturales » individualisées que prônait Le Corbusier. Quant à la déambulation du visiteur, il n'y avait pas à proprement parler de signalétique directionnelle au sein de 9o', car le dispositif était directif en soi. Mise à part une flèche indiquant à l'entrée le sens de la visite et les numéros affichés au départ de chaque galerie, les visiteurs, une fois embarqués dans le dispositif, suivaient spontanément la diagonale qui les menait d'une galerie à l'autre. Rarement, ils revenaient en arrière, bien qu'ils fussent parfaitement libres de le faire.

\section{Pouvaient-ils néanmoins retourner par l'extérieur?}

Tout à fait. En se promenant dans les galeries, le visiteur avait la possibilité d'en sortir par le côté nord qui était ouvert. Dans ce cas, il sortait du dispositif et donc de la scénographie. Il pouvait alors continuer à regarder l'exposition à distance en déambulant dans l'allée nord (fig. 4). Ce passage de la scénographie à l'espace réel est un aspect important du projet. 
La déambulation a en effet été au centre d'un certain nombre de réflexions importantes au début du $20^{\mathrm{e}}$ siècle qui semblent avoir disparu, détrônées par des impératifs économiques ou écartées en raison de l'impact trop fort et séduisant du white cube. Olivier Lugon, dans un article fort intéressant ${ }^{5}$, précise avec justesse que le point commun à tout type d'expositions (commerciales ou non), c'est la nécessité d'une déambulation. Mais ce paramètre de la visite lui semble le plus souvent ignoré dans les expositions artistiques dont le mode de fonctionnement reste, aujourd'hui encore, proche du principe du magasin: présenter au mieux un produit. À la fin de son article, Lugon conclut que le cheminement le plus directif n’est pas forcément le plus autoritaire. Cette remarque m’a intéressée car dans le cadre de 9o', je voulais un dispositif qui, sans résoudre ce dilemme, mette en avant cette ambivalence : liberté et contrainte. D'un côté, un dispositif rigoureux, systématique et presque autoritaire avec sa diagonale, ses galeries parallèles, ses horloges de hall de gare et des règles strictes pour chacun des accrochages et, de l'autre, une structure fluide et ouverte qui donne au visiteur une certaine liberté de mouvement, lui offrant même la possibilité de sortir de l'espace d'exposition sans qu'il puisse, il est vrai, totalement y échapper!

\section{temps de l'ceuvre, de la collection, de l'espace}

Les douze espaces qui sillonnent ce dispositif obéissent à des concepts directeurs différents et varient les formats d'exposition, depuis les modalités d'accrochage jusqu'aux "paratextes» (cartels, titres, panneaux explicatifs). Comment s'enchaînaient les salles et quelles étaient leurs particularités respectives?

En répondant à l'appel du Frac Franche-Comté, j’ai opté pour un projet qui ne consisterait plus à montrer une sélection subjective d'œuvres autonomes issues de la collection mais la collection comme un tout, une entité. Le système de dix galeries parallèles proposées aidait en cela: ces couloirs de longueur identique avaient pour fonction de présenter la collection sous forme d'un panel représentatif. Pour mettre en place cette "présentation ", j’ai alors défini, à partir de la notion de temps, des règles strictes d'accrochage dans chacune des dix galeries. Trois types de temporalités conditionnaient ces règles: 1 ) le temps de la collection; 2) le temps de l'œuvre d'art; 3) le temps de l'espace. Ces systèmes temporels

5. Olivier Lugon, «Des cheminements de pensée. La gestion de la circulation dans les expositions didactiques », Art Press spécial, n 21 «Oublier l'exposition », janvier 2000, p. 17 . 
étaient essentiels puisque, grâce à eux, j’allais pouvoir évacuer toute approche thématique.

Les paratextes ${ }^{6}$ (cartels, panneaux d'explication, éclairages et autres éléments qui constituent et orientent le parcours) avaient une place de premier plan. À travers eux, je ne cherchais pas à créer une unité, une cohérence ni un récit, mais à faire ressortir des différences pour mieux permettre aux visiteurs de prendre conscience des multiplicités de lectures possibles d'une œuvre. Ces distinctions, sans doute peu analysables au premier abord, étaient signifiées dans un dépliant proposé au visiteur à l'entrée de l'exposition. À la fois mode d'emploi et manifeste, ce document était primordial pour comprendre les systèmes à l'origine des accrochages. Mais il n'était pas indispensable: on pouvait aborder l'exposition sans directive, comme une balade.

Le «temps de la collection» était sans doute l'approche la plus pragmatique. Elle consistait à interroger les diverses temporalités à l'œuvre dans la collection du Frac (année de sa création, dates des œuvres, années des acquisitions...). Par exemple, la galerie intitulée 1960-200o n'exposait que l'œuvre la plus récente et l'œuvre la plus ancienne de la collection, à savoir une peinture d'Alfred Courmes datée de 1960 et une «sculpture» du jeune artiste Julien Berthier datée de 2008 . Cette confrontation permettait au visiteur d'aborder ces deux œuvres pas seulement pour elles-mêmes, mais en fonction d'un raccourci chronologique radical qui le renvoyait à l'histoire du Frac.

Autre exemple, la galerie 1984: comme son titre l'indique, elle regroupait l'ensemble des œuvres de l'année 1984, sans exception. J'avais constaté qu'au sein de la collection, l'année 1984 rassemblait le plus grand nombre d'œuvres: cinquante-huit, alors que la moyenne des acquisitions était de vingt à trente $œ u v r e s$ par an pour les périodes fastes. Dans les faits, cette date correspondait à la mise en fonction réelle du Frac qui bénéficiait des subventions accumulées depuis sa création en 1982. Pour «contextualiser» l'accrochage, j'avais opté pour un panneau d'exposition réunissant des extraits de journaux datant de la même période. Ces articles, qui décrivaient souvent avec humour et/ou agacement les œuvres acquises par le Frac, contribuaient à replonger le visiteur dans le climat de l'époque. À cela s'ajoutait un système d'éclairage particulier à partir de lampes dites «rétroviseurs» qui projetaient sur les œuvres un halo plutôt jaune:

6. Ce terme est notamment employé par Michel Gauthier dans son texte «Dérives périphériques», Les cahiers du Musée national d'art moderne, n 56-57 «Du catalogue ", 1996, p. 130. L'auteur se réfère ici au livre de Gérard Genette, Seuils, Paris, Éditions du Seuil, coll. «Poétique», 1987. 
un modèle très caractéristique de cette période. La petitesse de l'espace ne permettant pas d'exposer l'ensemble de ces œuvres, j’en avais entreposé une partie dans un rack disposé au bout de la galerie. Ces ouvres en attente étaient destinées à renouveler l'accrochage durant l'exposition.

Une des conséquences singulières qu'impliquait ce système d'exposition était de m’obliger à reléguer mes goûts de commissaire au second plan. De manière théorique, j’ajouterais que cette démarche renvoyait à une approche «rieglienne» de l'art qui m'est proche: à savoir que toutes les œuvres sont issues d'un esprit du temps et d'un contexte culturel particulier et, de ce point de vue, sont intéressantes.

La notion de «temps de l'œuvre» donnait lieu à des accrochages, je dirais, plus formels, telle la galerie intitulée Temps Séquence, dédiée exclusivement aux vidéos de la collection. Au lieu, cependant, d'apparaître par thème, les vidéos étaient regroupées en fonction de leur durée (cinq minutes, dix minutes et une heure) et projetées sur trois écrans disposés en parallèle dans la galerie. L'accrochage mettait ainsi en évidence l'influence du temps dans la perception d'un film. Par ailleurs, cette classification excluait toutes les vidéos de durée différente, ce qui m’obligeait, de nouveau, à faire fi de mes propres choix.

Un autre espace répondant à cette notion était la galerie Le temps du récit qui ne montrait que les œuvres pensées en séries ou en séquences. Encore une fois, le format déterminait l'accrochage. Pour souligner l'importance des séries dans la pratique artistique, celles-ci étaient accrochées de manière rigoureusement horizontale, de sorte que l'on ne percevait tout d'abord qu'un ensemble de frises. À travers cet accrochage extrêmement graphique, je voulais signifier qu'un format similaire (la série) offrait des enjeux et contenus artistiques extrêmement variés. Ainsi, les dessins rudimentaires de Claude Closky ou les broderies «féministes » d'Annette Messager n'avaient effectivement rien à voir avec les conceptions plus cinématographiques d'un Hans Schabus ou d'un Loïc Raguénès, ou les saynètes d'un Bernard Faucon.

Temps de la collection, temps de l'œuvre: ces concepts directeurs ont une résonance familière dans le contexte d'une exposition d'art. Il n'en va pas de même pour «le temps de l'espace»: pourquoi ce choix?

C'était sans aucun doute la notion la plus «abstraite» et, paradoxalement, la plus concrète. À travers elle, j’abordais l'idée de mise en scène. Cela donna lieu à l'espace le plus spectaculaire de l'exposition : La Grande Galerie (fig. 5)... 


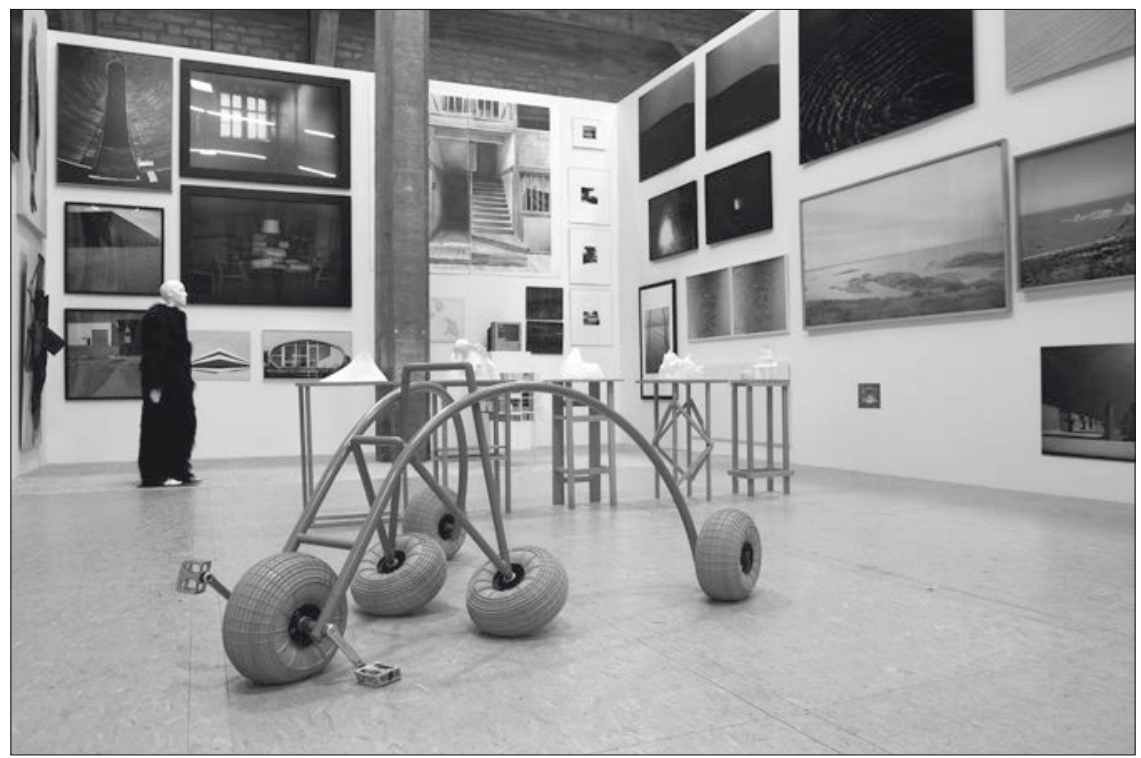

Fig. 5: La Grande Galerie. Photographie Aurélien Mole.

\section{... en référence à la Grande Galerie du Louvre?}

Absolument. Pour m’approcher au plus près de cette évocation des Grandes Galeries de Peintures mises en place aux $18^{\mathrm{e}}$ et $19^{\mathrm{e}}$ siècles, j’avais décidé de doubler le volume de la galerie. Ici, les murs montaient soudain à cinq mètres de hauteur et s'élargissaient à près de huit mètres. J'avais également emprunté le principe d'accrochage «en tapisserie», longtemps refoulé, voire banni des musées d'art moderne et contemporain, afin de questionner le sens et l'intérêt qu'il pouvait présenter aujourd'hui. Peut-être qu'en vue des nouvelles expériences visuelles qui ont fait leur apparition dans notre champ de perception, tels les écrans Internet recouverts d'icônes, pouvons-nous être à nouveau réceptifs à ce genre de disposition. Cet accrochage m'intéressait également car il imposait soudainement au visiteur une attitude différente. À l'inverse des autres espaces qui exigeaient une certaine mobilité, le visiteur pouvait ici rester à un point fixe pour percevoir l'ensemble, mais il lui fallait relever et baisser la tête pour percevoir chacune des œuvres. La présence d'un grand cartel unique sur lequel figuraient les titres des œuvres et leur emplacement aidait en ce sens. Le visiteur pouvait alors avoir le sentiment de se retrouver au milieu d'un immense tableau où chaque œuvre était partie d'un tout. Pour rendre l'ensemble plus harmonieux et en faciliter la lecture, 
j'avais regroupé les travaux par thème (les formes abstraites, la nature humaine, les phénomènes naturels, la nature catastrophique, les structures architecturées) et par correspondance esthétique (couleurs, noir et blanc, nuances de bleu...). En procédant ainsi, j’avais également en tête l'Atlas Mnémosyne d'Aby Warburg?. À l'instar de ce système fascinant qui agençait ensemble des images issues de domaines les plus divers, je souhaitais, à partir de la collection, composer un accrochage qui évoque une sorte d'atlas d'images.

L'accrochage en tapisserie était «mis à jour» par l'Atlas de Warburg, en quelque sorte?

Exactement, revu à travers le prisme de Warburg. Il aurait été vain et fastidieux de chercher à restituer les logiques qui, au $18^{\mathrm{e}}$ et au $19^{\mathrm{e}}$ siècle, structuraient ces accrochages. Ce type d'installation doit au contraire se nourrir des expériences qui, tout au long du $20^{\mathrm{e}}$ siècle jusqu'à aujourd'hui, ont enrichi notre perception des images et de l'art.

Est-ce que les galeries Period Room et Lobby rejoignaient, elles aussi, cette conception du temps structuré par un modèle spatial donné?

En effet. Le terme period room m'est cher pour plusieurs raisons. Historiquement, c'est sans doute l'un des systèmes de présentation muséale les plus anciens ${ }^{8}$. Lautre raison a trait à l'omniprésence du modèle de l'espace reconstitué dans

7. Le Bilderatlas Mnemosyne qu'Aby Warburg systématise à partir de 1928 demeure inachevé à sa mort en 1929. La version de référence, de 1929, comporte 63 panneaux de reproductions photographiques. Il a été récemment republié dans l'édition allemande des œuvres complètes de Warburg: Aby Warburg, Gesammelte Schriften. Studienausgabe, Bd. II 1.2: Der Bilderatlas MNEMOSYNE, édition dirigée par Martin Warnke assisté de Claudia Brink, $2^{e}$ éd. revue et corrigée, Berlin, Akademie Verlag, 2003.

8. L'Hôtel de Cluny inauguré par Alexandre du Sommerard en 1832 (actuel Musée du Moyen Âge) peut être vu comme une des premières Period rooms, puisqu'il replaçait les objets exposés dans leurs environnements «organiques» reconstitués. Stephen Bann oppose ce modèle, relevant du modèle de la synecdoque, au principe de la métonymie sous-jacent au Musée des monuments français d'Alexandre Lenoir (Paris, Couvent des Petits-Augustins, 1795-1816), centré sur la reconstruction de fragments-spécimens. Voir Stephen Bann, "Poetics of the Museum: Lenoir and Du Sommerard», The Clothing of Clio: A Study of the Representation of the History in Nineteenth-Century Britain and France, Cambridge, Cambridge University Press, 1984, p. 77-92. Voir également à ce propos la section «Les débuts des musées et le cas des period rooms», du livre de Jérôme 


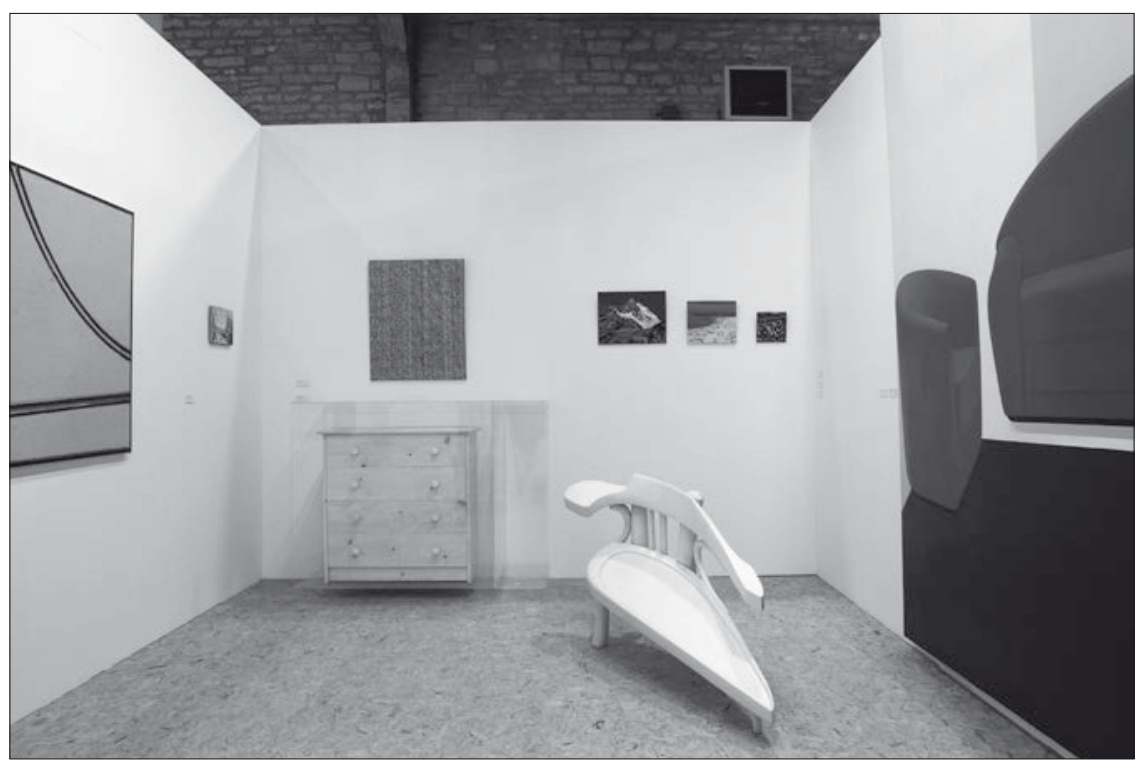

Fig. 6: Period Room. Photographie Aurélien Mole.

nos sociétés de consommation. Cela va du plateau télévisuel ou de cinéma en passant par les showrooms, les resorts tels Celebration en Floride ou La vallée des marques à Marne-la-Vallée jusqu'au Mont-Saint-Michel ${ }^{9}$. Il y a peu de différences, je pense, entre la visite des plateaux de tournage et le fait de se rendre chez Ikea: on y va pour voir des reconstitutions et les «consommer». C'est une situation fascinante. Elle révèle des enjeux esthétiques concernant le rapport entre l'image, le lieu et la fiction auxquels il faut réfléchir.

Dans le cadre de 9o', la Period room consistait à reconstituer un appartement à partir d'œuvres d'art (fig. 6). J'avais en effet remarqué qu'au sein de la collection, un grand nombre d'œuvres évoquaient le quotidien : une commode (Didier Marcel), une garde-robe (Wiebke Siem), un pull (Erwin Wurm), un lit (Matthew McCaslin), un verre (Markus Raetz), des plantes, une chaise (Philippe Cogné), un balai (Régis Perray)... Il n’était nullement question de créer un décor contemporain pour intégrer ces œuvres. Les œuvres devaient créer le décor. En opérant

Glicenstein, L'art: une histoire d'expositions, Paris, Les Presses universitaires de France, coll. «Lignes d'art», 2009, p. 18-29.

9. Dans son essai «Voyage dans l'hyperréalité», Umberto Eco recense de manière fascinante l'ensemble de ces lieux. Voir La guerre du faux [1985], trad. Myriam Tanant avec la collaboration de Piero Caracciolo, Paris, Grasset, 2008, p. 7-93. 
ce déplacement par rapport au principe de la Period room, ces œuvres donnaient une vision déformée de la réalité et du temps qui n’était pas sans rappeler le livre de Lewis Carroll, Alice's Adventures in Wonderland (Alice au pays des merveilles, 1865), un ouvrage extrêmement contemporain dans ses glissements entre réalité fictive et fiction de réalité.

Dans la galerie Lobby, le titre avait également un rôle déterminant. Il créait le lieu et conditionnait notre appréhension de l'espace. À l'origine, le mot anglais lobby signifie « couloir». Pris dans ce sens général, il résumait parfaitement la manière dont je voulais que les dix galeries de l'exposition fonctionnent: à la fois comme lieux de passage et comme lieux d'exposition. D'autre part, les lobbys sont ces espaces que l'on trouve habituellement à l'entrée des immeubles de standing, à la fois salle d'attente et lieu de transit, dotés de confortables sofas et souvent d'œuvres d'art remarquables. La galerie Lobby était conçue à leur image. Ses deux canapés invitaient enfin le visiteur à s'asseoir et à suspendre son temps de parcours. Les œuvres retenues pour cet espace l'étaient en fonction de leur facture résolument graphique ou décorative. À l'extrémité de cette galerie, quatre gros classeurs disposés sur des tables basses offraient, en fin de parcours, la possibilité pour le visiteur de visualiser les œuvres non présentées dans l'exposition.

C'était la Galerie des œuvres absentes?...

... absolument, chaque œuvre y figurait sous forme de photo au format $\mathrm{A}_{4}$ avec, au verso, une fiche technique. J'avais, en fait, repris le mode de classement qu'utilise habituellement la documentaliste du Frac. En feuilletant ces classeurs, le visiteur averti pouvait rapidement se rendre compte qu'il ne s'agissait pas d'un «classement des refusés». On y trouvait des œuvres importantes de Bertrand Lavier, de Christian Boltanski, de Marina Abramovic qui n'avaient pu être montrées au sein de l'exposition faute de place, mais surtout parce que mon système ne les réclamait pas.

Cette présence de fiches permettait donc, encore une fois, de repenser la notion de «collection $» ?$

Tout à fait. Ces fiches standard étaient pour moi fondamentales. Lors d'expositions sur les collections, les œuvres non choisies sont le plus souvent reléguées aux oubliettes. Elles n'existent plus aux yeux du commissaire, encore moins aux yeux du visiteur. Dans le cas de 9o', je voulais justement que les œuvres 
non sélectionnées soient prises en considération, car il s'agissait ici d’aborder la collection comme un ensemble, une entité.

\section{EXPOSER L'EXPOSITION}

9o' expose la collection sous différents aspects: son entretien, le fonctionnement de ses archives, son élaboration historique...

Oui, c'est quasiment une approche anthropologique.

Les différents modes d'exposition activés au sein de 9o' viennent déconstruire le mythe de la collection homogène, logique, rationnelle, exhaustive.

En décidant d'aborder la collection comme un ensemble, je ne me laissais aucune échappatoire. Toutes les œuvres devaient être regardées, analysées, quelles que soient mes préférences. Les systèmes que j’avais établis allaient déterminer en priorité les choix. Aborder la collection comme un tout est une expérience étonnante. On comprend alors qu'aucune acquisition n'est le fruit du hasard: toutes sont le résultat d'un choix collectif, d'orientations esthétiques précises. Elles sont le reflet d'une politique culturelle singulière, celle des Frac. Tout cela forme une histoire passionnante qui a un sens. J'entends souvent dire que les collections des Frac sont irrégulières, inégales. Mais la plupart des collections d'art contemporain sont inégales et irrégulières, heureusement! Le but des Frac est d'acheter de l'art contemporain avec parfois des moyens modestes. Cette politique donne peu de recul et donc le risque de passer à côté d'œuvres majeures est évident. À cela s'ajoutent les phénomènes de mode auxquels peu d'entre nous échappent. La plupart des grands musées donnent une représentation universaliste de la collection comme une somme de chefs-d'œuvre. Au contraire, il me semblait enrichissant de ne pas dissimuler les particularismes d'une collection.

La multiplicité des lectures et des pistes d'interprétation proposées au sein de 9o' traduit-elle un certain constat d'échec des expositions à discours unique, très prononcé, "signé»? Ou bien, vise-t-elle davantage à mettre en évidence l'exposition comme discours visuel et spatiotemporel et à lutter contre la prétendue neutralité de l'acte d'exposer?

Cette exposition ne souligne pas tant l'échec d'une pensée que le fait que l'on soit peut-être arrivé au bout d'un système. 90' tente de mettre à plat un 
ensemble de principes qui existent depuis le $18^{\mathrm{e}}$ siècle, pour éventuellement les dépasser. Avec ses systèmes d'accrochage, son dispositif, sa scénographie, son titre, ce projet cherche à réfléchir autrement le principe de l'exposition en esquissant d'autres rapports à l'espace, au public et aux œuvres. Pour cela, j'avais décidé de ne plus mettre au centre l'œuvre mais les principes même d'exposition. Au lieu de dissimuler - comme cela est traditionnellement le cas - les éléments expographiques (cartel, éclairages, support, accrochage, etc.) - pour mieux révéler l'œuvre, j'ai au contraire tenté de les rendre visibles pour montrer à quel point ces éléments orientent notre lecture et donc notre réception des œuvres.

En ce sens, je me sens très proche des théories et concepts développés par Jacques Hainard au Musée d'ethnographie de Neuchâtel. L'ancien directeur de ce musée suisse a émis le concept singulier d'une «muséographie de la rupture ${ }^{10}$ ». Ce principe propose au conservateur de ne plus être l'esclave de l'objet mais de rendre l'objet esclave du conservateur. Il ne s'agit plus d'instrumentaliser l'objet mais de réfléchir à son instrumentalisation. Cela pourrait contribuer à libérer notre regard et par là même, l'œuvre. Ce n'est pas une affirmation mais une piste de réflexion à expérimenter... Peut-être arriverons-nous un jour à sortir du système, plus dominant que jamais, du white cube et de l'emprise de la thématique. Ce jour-là, sans doute, devrons-nous penser le domaine des arts plastiques autrement.

L'historien de l'art Hal Foster décrivait l'importance d'une attitude réflexive dans les pratiques artistiques récentes dans ces termes: "cadrer le cadreur au moment même où il cadre l'autre"l ". Le parti pris de l'exposition 9o' n'est-il pas similaire : être commissaire d'exposition, organiser, superviser, inventer l'exposition, mais en même temps exposer l'acte d'exposition?

Absolument. L'important étant cependant de savoir comment et pourquoi on décide d'exposer l'acte d'exposition! Dans le cas de l'exposition 9o', l'idée était de rendre visibles les conditionnements possibles des œuvres au sein d'un parcours pour interroger le sens même de l'exposition. Quels types d'explications,

10. Jacques Hainard, «Du musée spectacle à la muséographie de la rupture », dans Marc-Olivier Gonseth, Jacques Hainard et Roland Kaehr (dir.), Cent ans d'ethnographie sur la colline de Saint-Nicolas 1904-2004, Neuchâtel, Musée d'ethnographie, 2005, p. 367-374.

11. Hal Foster définit cette attitude comme un «travail de parallaxe». Voir son «Portrait de l'artiste en ethnographe», Le retour du réel. Situation actuelle de l'avantgarde, trad. Yves Cantraine, Frank Pierobon et Daniel Vander Gucht, Bruxelles, La lettre volée, 2005, p. 247 (les italiques sont de l'auteur). 
quels types d'éclairages pour quels effets et quelles orientations esthétiques? La méthode employée était la différenciation, le contraste. Ainsi, dans la galerie Chronologie, j'avais décidé d'accompagner chaque œuvre exposée d'un long cartel expliquant les raisons de leur acquisition. Ce parti pris m'avait conduite à interviewer au préalable les directeurs successifs que furent François Cheval et Anne Dary à ce sujet. Ces entrevues extrêmement vivantes et très simples étaient ensuite retranscrites, en partie, sur ces longues fiches. Le visiteur pouvait ainsi découvrir les coulisses des Frac: comment se font ces acquisitions, ce qu'est un comité technique, la raison de ce choix...

Par souci de rupture et de contraste, la galerie suivante, "Sans titre », n'avait aucun cartel, aucun titre, rien. À l'instar de la galerie Chronologie, le principe consistait ici à exposer une dizaine d'œuvres réalisées à des dates très variées. Mais au lieu de suivre une évolution chronologique, les œuvres étaient présentées selon un thème que j’avais déterminé, «la ligne d'horizon », et qui privilégiait des rapprochements de forme. L'espace devenait ainsi délibérément subjectif. Afin d'accentuer le caractère fortement esthétique de cette «composition », j'avais remplacé les grands néons à éclairage diffus et régulier par des spots plus recentrés. L'espace ainsi mis en scène avait un aspect sensiblement plus théâtral.

Le visiteur passait donc d'un espace chargé de références avec des cartels parfois aussi grands que les œuvres, des dates inscrites en gros à même le sol, à un espace dépourvu de tout repère, à l'exception d'une feuille discrètement mise à disposition à l'entrée de la galerie sur laquelle figuraient les titres et emplacements des œuvres.

Cette pratique de l'exposition, sans titres ni cartels explicatifs, qui rejoint la conception de Heinrich Wölfflin d'une histoire de l'art "sans noms », a également soutenu le modèle du white cube, par exemple dans les premières expositions d'Alfred Barr au MoMA de New York; les accrochages de la collection permanente et d'expositions telles que Cubism and Abstract Art (1936), ou Fantastic Art, Dada, Surrealism (1937) reposaient justement très souvent sur des rapprochements formels pour faire ressortir l'évolution d'un style, une école, un esprit de mouvement...

Pour moi, l'absence de cartel sert souvent à «scénariser » davantage l'espace ou à renforcer la parenté avec le salon, l'habitat privé. Cela dit, je ne suis pas convaincue que l'absence de cartel dans une salle de musée permette au visiteur de mieux saisir une école ou un mouvement. À mon sens, c'est une approche bourgeoise de l'art. 


\section{INTERMÉDIALITÉ DE L’EXPOSITION}

Le plan de 9o' évoque des associations intermédiatiques: on aurait envie de parler de séquences (de galeries) qui se déroulent à la manière d'un film: marquées par un rythme, impliquant des procédés de montage (de coupe), avec des points d'arrêt mais aussi de continuité. Plusieurs chercheurs ont fait ressortir des similarités entre exposition et cinéma, particulièrement lorsque l'exposition devient, pour reprendre la formule d'Olivier Lugon, un "médium de masse" ${ }^{12}$. Ce souci me semble avoir joué un rôle dans 9o', d'autant plus que quatre-vingt-dix minutes est la durée moyenne d'un film. Y aurait-il, à votre avis, une intermédialité fondamentale dans l'exposition, si l'on comprend l'intermédialité comme l'idée qu'un médium n'est jamais seul, qu'il contient toujours en puissance la mémoire de et la relation à d'autres médias (le cinéma qui comporte le théâtre, le vaudeville, la photographie, la foire)? Quelle était la place du cinéma, des procédés cinématographiques, dans l'expérience que l'on pouvait faire de 9o'? Plus généralement aussi, pour sortir des approches ancrées dans les œuvres, il faudrait peut-être reconnaître qu'il y a une intermédialité de l'exposition comme médium?

En imaginant le dispositif de 9o', j’espérais effectivement pouvoir pointer une certaine proximité entre le principe de l'exposition, le cinéma et le théâtre. Ce sont trois domaines très proches mais dont «l'intermédialité » est, selon moi, peu traitée par les commissaires ou de manière parfois trop littérale. Un des liens qui unit ces trois domaines est sans aucun doute la fiction. En tant que mode de présentation et de représentation, l'exposition, le cinéma et le théâtre forment trois espaces fictionnels et fictifs. C'est cet aspect que j'ai tenté d'aborder au sein du projet. Concrètement, cela a donné lieu à une sorte de rupture entre espace réel et espace expographique. En pénétrant dans la première galerie, le visiteur se retrouvait tout d'abord immergé dans l'espace d'exposition. Puis en progressant de galerie en galerie, la diagonale l'amenait irrésistiblement vers le côté ouvert du dispositif, tant et si bien qu’arrivé à l'avant-dernière galerie, il lui suffisait d'un pas pour sortir de l'espace scénographié et investir l'espace réel. Ce passage lui permettait de prendre conscience de la nature fictive, éphémère, voire décorative, du dispositif de l'exposition. L'espace réel formait, quant à lui, une longue allée que le visiteur pouvait parcourir. Il effectuait alors une sorte de travelling qui lui donnait à voir les galeries comme une suite de plateaux de tournage ou de décors

12. Olivier Lugon, «La photographie mise en espace: les expositions didactiques allemandes (1925-1945)», Études photographiques, n 5 , novembre 1998, p. 97-118; voir aussi Lugon, 2000. 
de théâtre. J'ai d'ailleurs voulu exploiter ce rapprochement pour la publication qui fera suite à cette exposition en demandant à un artiste de filmer en travelling l'exposition à partir de cette allée nord.

Les analogies entre cinéma et exposition ont, en effet, été relevées et étudiées dès le début du $20^{\mathrm{e}}$ siècle. Ce fut même le fil directeur pour les scénographies «dirigistes» d'Herbert Bayer, pour qui l'exposition s'apparentait à un film renversé: au lieu de visualiser d'un point fixe des images mobiles, l'espace d'exposition invitait le visiteur à bouger devant des images fixes. Bayer avait même imaginé un tapis roulant pour «guider» le visiteur dans ses scénographies $^{13}$. Cette idée de la mobilité du visiteur était, certes, centrale pour moi. Cependant les rapprochements que je voulais mettre en avant étaient moins d'ordre structurel que fictionnel. Dans quel type de fiction évolue-t-on? Quel sens cela peut-il avoir? En fait, ce qui m'importait résidait davantage dans cette idée de franchissement, ce passage entre décor et espace réel qui offrait soudain un niveau de lecture différent.

\section{DOCUMENTER : UNE PENSÉE CRITIQUe PAR L'iMAge}

La documentation de l'exposition tient une place spéciale dans ce projet. Ce parti pris m'intéresse beaucoup puisque la documentation visuelle devient un enjeu majeur du domaine en pleine émergence des études de l'exposition, en particulier en ce qui concerne l'histoire des expositions passées. Dans certains cas, elle pose problème par sa rareté, voire son absence; lorsqu'elle existe, les choix de documentation adoptés ont une importance cruciale (photographie noir et blanc plutôt que couleur; choix d'angle de prise de vue; sélectivité de la prise de vue). Un cas exemplaire est la vue de la salle des ceuvres suprématistes de Kazimir Malevitch lors de La dernière exposition futuriste de tableaux o,10 (zéro-dix), tenue en 19151916 à Petrograd. En exagérant à peine, on pourrait clamer que son succès tient en partie au fait même que cette photographie fut prise alors que nous ignorons presque tout des autres espaces de cette exposition collective, voire du hors-champ de cette salle. Cette vue témoigne également des débuts historiques de la pratique de documentation photographique. Cinquante ans plus tard, Brian O'Doherty critiquera la standardisation de cette pratique de l'installation shot, réduite le plus souvent au plan général d'une portion de salle d'exposition dépourvue de tout spectateur. Pour lui, ce type de documentation est un des piliers symboliques de la logique du white cube ${ }^{14}$.

13. Olivier Lugon rapporte cette idée de Bayer, non réalisée. Voir Lugon, 2000, p. 20. 14. O'Doherty, 2008, p. 37. 
Dans le cas de 9o', la documentation de l'exposition a donné lieu à un travail de collaboration avec différents artistes. Qu'est-ce qui a motivé ce choix de multiples stratégies de documentation?

Je voulais développer une réflexion sur le contenu, le sens et la place de l'image au sein d'un catalogue. Pour ce faire, j'ai fait appel à différents intervenants, dont la démarche artistique pouvait livrer un regard original et différencié sur l'exposition. Marina Gadonneix m’a proposée de prendre des photos de nuit de 9o', mettant en avant les œuvres et la structure qui les montre. J'ai demandé à Charles Frégé d'intégrer le visiteur dans ses clichés; il a décidé d'inclure aussi son assistant ainsi que tout le matériel de prise de vue. À travers ces éléments supplémentaires, ses photos mettent soudain en exergue ces glissements entre l'espace d'exposition, le plateau de cinéma et la scène de théâtre.

Les intervenants artistes auxquels je me suis adressée ne sont pas tous des photographes. Je voulais éviter que la photo soit le seul média capable de documenter l'exposition. Il y aura également un film tourné par l'artiste allemand Rainer Oldendorf et des dessins réalisés par le jeune plasticien Julien Berthier.

\section{Y aura-t-il des photographies de cimaises plus classiques?}

J'ai effectivement demandé à Aurélien Mole, un photographe rôdé aux techniques de la photographie d'exposition, d'effectuer des prises de vue systématiques de chacune des galeries. Je souhaitais, à partir de ces documents, reconstituer cimaise par cimaise l'ensemble de l'exposition. Il faut souligner que l'agencement parallèle des murs des galeries rappelait les pages d'un livre. Ce sentiment pouvait être renforcé par leur numérotation systématique de un à onze qui, inévitablement, renvoyait au système de pagination. Le dispositif d'exposition pouvait être ainsi perçu comme une sorte de livre «en trois dimensions».

El Lissitzky avait une conception similaire. Ses livres expérimentaux devaient matérialiser son utopie de l'objet mobile et du lecteur physiquement impliqué. C'étaient des objets à manipuler, et non seulement à lire: par exemple, grâce à une organisation sous forme d'index prédécoupés ${ }^{15}$. Benjamin Buchloh fut l'un des

15. Voir, par exemple, le recueil de poèmes de Vladimir Maïakovski mis en page par le «constructeur de livres» El Lissitzky: Pour la voix (Dlia golosa). Publié en 1923, il fut édité en fac-similé et traduit en anglais par Patricia Railing (dir.), For the voice, Cambridge (Mass.), MIT Press, 2000. 
premiers à rapprocher le travail de Lissitzky sur les livres de celui, ultérieur de quelques années, portant sur l'espace d'exposition ${ }^{16}$.

Lissitzky voulait en effet stimuler le visiteur par des expositions vivantes qui l'impliquent. De mon côté, je ne cherchais pas forcément à projeter le visiteur dans un espace qui lui rappelait le livre. Ce qui m'intéressait, c'était de le confronter à un fonctionnement, à des modes rigoureux de classification. Si le dispositif s'apparentait à un livre, c'est parce qu'il tentait d'être aussi systémique que peut l'être un catalogue. Ce sont les systèmes de classement, les logiques de pensée qui m'intéressent et pas forcément les ressemblances structurelles. En cela, ma démarche est assez différente de celle d'Alexandre Dorner qui, lors du réagencement de la collection du musée municipal de Hanovre vers 1925, avait, semble-t-il, cherché à créer une analogie entre les espaces d'exposition et le livre ${ }^{17}$. Il voulait scénographier un espace comme on «scénographie» une histoire dans un livre, en «salles-chapitres», avec un début marqué par une introduction et une fin signifiée par une conclusion. J'ai pris connaissance de cette expérience après avoir monté 9o'. Cette découverte me réjouissait car la proximité entre la structure de l'exposition et le livre m’est apparue au cours du montage. À l'inverse de Dorner, mon dispositif ne mettait pas tant en avant une narration que des systèmes. Actuellement, je réfléchis à la façon dont je vais «traduire» ces glissements au sein du catalogue. Les photos systématiques de Mole offrent une réflexion en ce sens: mises bout à bout, elles donnent à voir l'exposition comme un catalogue raisonné en trois dimensions. À ces clichés s'ajoutent des prises de vue réalisées par l'artiste Frédéric Lefever une fois l'exposition démontée : ces images presque abstraites laissent transparaître les traces des clous et coups de crayon qui, bien sûr, évoquent la page blanche.

Cette publication - le catalogue - sera donc un prolongement de l'exposition $90^{\prime}$.

Le catalogue sera en fait un ouvrage indépendant dans lequel l'exposition ne représente qu'une partie. Ce n'est donc pas un catalogue classique d'exposition qui répond aux logiques urgentistes du vernissage, mais plutôt un projet à long

16. Voir Benjamin H.-D. Buchloh, «Faktura et factographie» [1984], Essais historiques I - Art moderne, trad. Claude Gintz, Villeurbanne, Art édition, 1992, p. 65-126.

17. Analogie relevée par Glicenstein, 2009, p. 130 (source non citée). 
terme qui, dans l'approche, s'apparente davantage à un catalogue raisonné de la collection. Pour le dire vite, cet ouvrage proposera une analyse de la collection à travers ses deux principaux médiums que sont l'exposition et le catalogue.

En abordant le catalogue comme un médium, je souhaite en effet développer un corpus critique par l'image. Pourquoi seraient-ce inévitablement les textes qui devraient nous éclairer quant aux œuvres et à leur mise en espace? Ce projet éditorial m’a amenée à me pencher sur l'histoire du catalogue dont l'histoire, l'origine et le contenu ont été peu étudiés ${ }^{18}$. L'illustration, sa nature (dessin, gravure, photo couleur ou noir et blanc, etc.), son style, son emplacement semblent ignorés: on se concentre plus volontiers sur les textes (notices, légendes, etc.). On se retrouve aujourd'hui face à une pléthore de catalogues d'art mais aucune réflexion de fond ne semble être menée sur l'histoire, l'origine et le contenu de ces ouvrages. Comment, au cours des siècles, les œuvres étaient classées, rangées, mises en image, mises en page, bref cataloguées? Et que cela signifie-t-il dans notre rapport à l'art?

Le catalogue comptera trois parties: la première consistera à montrer la collection dans le contexte de l'exposition 9o'; la deuxième, dans le contexte de l'institution qu'est le Frac Franche-Comté. Cette deuxième section se concentrera sur les œuvres non exposées lors de 9o', soit près de la moitié (44\%) et s'attachera à montrer les œuvres non plus en plan serré, mais dans leur contexte de monstration de l'époque. Elle rassemblera également l'ensemble des cartons et affiches qui, au fil des ans, ont accompagné ces expositions, contribuant à créer l'identité visuelle du Frac. Le travail de recherche mené dans les archives du Frac m'a permis de retrouver et d'identifier des photographies étonnantes d'expositions organisées dans des compagnies d'assurances, des supermarchés et même des trains! Ces documents reflètent la politique tout terrain des Frac lors de leur lancement. Il serait d'autant plus opportun d'étudier le recours à ces sites qu'aujourd'hui des commissaires audacieux, tel Moritz Kung ${ }^{19}$ en Belgique, axent leur pratique curatoriale sur l'utilisation de lieux d'exposition autres que les musées et centres d'art. Le catalogue mettra en valeur cet aspect trop souvent ignoré.

18. Situation que déplore déjà en 1996 Jean-Pierre Criqui dans son éditorial consacré au catalogue dans Les cahiers du Musée national d'art moderne, ${ }^{\circ}$ 56-57, «Du catalogue », 1996, p. 3 .

19. Responsable depuis 2002 du programme du DeSingel à Anvers et initiateur du projet «Curating the Library». 
La troisième partie regroupera l'ensemble des œuvres de la collection sous forme de miniatures reproduites à une même échelle et découpées suivant leur contour, formant ainsi une synthèse entre la première et la deuxième. J'aimerais, pour cette partie, développer une réflexion sur l'index, un système que je trouve passionnant parce qu'il peut régler, conditionner ou réorienter notre manière d'aborder, de lire un ouvrage. En général, on se sert de l'index sans réfléchir à son sens ni même à sa fonction ${ }^{20}$. Je voudrais essayer de mettre au point des portes d'entrée inattendues pour amener le lecteur à « déchiffrer » autrement cet ouvrage: ce pourraient être des entrées en fonction de la taille des œuvres, de pourcentages, de thématiques et pas forcément en termes d'ordre alphabétique, de noms d'artistes ou de pages. La publication est prévue pour la fin de 2010.

Dernière question, aussi provocatrice que banale, mais incontournable: comment vous positionnez-vous par rapport au débat sur l'exposition comme ouvre et le commissaire comme artiste?

Je trouve ce débat peu sérieux et surtout peu scientifique car, de ce que j’ai pu en percevoir, il fait souvent fi de toute l'histoire de la muséologie et des expositions. Surtout, il occulte le principal: le contenu des expositions. Qu'importe le statut du «faiseur", il me semble plus urgent de se pencher sur le statut et le devenir de l'exposition. Si l'artiste se sent menacé par le commissaire, c'est plutôt une bonne nouvelle pour l'artiste et le commissaire.

Vous ne vous considérez donc pas du tout comme artiste lorsque vous organisez une exposition?

Réfléchir comme cela n’a, selon moi, aucun intérêt. Qu'est-ce qui fait œuvre aujourd'hui et pourquoi? Voilà une question qui me semble plus pertinente. Quant à la question du commissaire-artiste ou de l'artiste-commissaire, elle doit s'inscrire dans un débat plus vaste sur le rôle et le statut de l'auteur.

20. Pour une réflexion sur la notion d'index, voir Patricia Falguières, «Les raisons du catalogue », Les cahiers du Musée national d'art moderne, n ${ }^{\circ 6-57 ~ " D u ~ c a t a l o g u e ~ », ~}$ 1996, p. 5-19. 
En même temps, vous revendiquez clairement le fait d'instrumentaliser les œuvres.

Dans son petit livre intitulé Qu'est-ce qu'un dispositif, Giorgio Agamben analyse de manière remarquable nos sociétés capitalistes ${ }^{21}$. Il explique comment ces dernières ont fini par générer des systèmes d'instrumentalisation extrêmement complexes et effrayants qui, tout en nous laissant le sentiment de les maîtriser, nous échappent totalement. Le monde de l'art n'est pas épargné par cette logique, bien au contraire. En effet, qui sert qui? Qui se sert de qui ? Les commissaires se servent des œuvres, les artistes se servent des commissaires. L'instrumentalisation est un sujet en soi qu'il ne faut pas éviter sous peine d'instrumentaliser encore un peu plus l'art et le visiteur.

21. Giorgio Agamben, Qu'est-ce qu'un dispositif?, trad. Martin Rueff, Paris, Payot \& Rivages, 2007 . 